\title{
Semi-global Robust Stabilization of A Class of Feedforward Systems
}

\author{
Minghui Zhu and Jie Huang
}

\begin{abstract}
This paper studies the robust stabilization of a class of nonlinear systems in feedforward form that is subject to both dynamic uncertainty and time-varying static uncertainty. For this purpose, a nonlinear small gain theorem with restrictions for time-varying nonlinear systems is established first. Then the small gain theorem is applied to achieve semiglobal robust stabilization for the class of feedforward systems in the presence of both dynamic and static uncertainties.
\end{abstract}

\section{INTRODUCTION}

In this paper, we will study the robust stabilization of nonlinear systems of the form:

$$
\begin{aligned}
\dot{x}_{i} & =c_{i-1} x_{i-1}+f_{i}\left(\xi_{1}, x_{1}, \ldots, \xi_{i-1}, x_{i-1}, \xi_{i}, u, d\right) \\
\dot{\xi}_{i} & =g_{i}\left(\xi_{1}, x_{1}, \ldots, \xi_{i-1}, x_{i-1}, \xi_{i}, u, d\right), \quad i=n, \cdots, 2 \\
\dot{x}_{1} & =C \xi_{1}+D u+f_{1}\left(\xi_{1}, u, d\right) \\
\dot{\xi}_{1} & =A \xi_{1}+B u+g_{1}\left(\xi_{1}, u, d\right), \quad t \geq t_{0} \geq 0
\end{aligned}
$$

where, for $i=1, \ldots, n, x_{i} \in \Re, d \in \Re^{n_{d}}, \xi_{i} \in \Re^{p_{i}}$, $u \in \Re, f_{i}$ and $g_{i}$ are globally defined $C^{1}$ functions satisfying $f_{i}(0, \cdots, 0, d)=0$ and $g_{i}(0, \cdots, 0, d)=0$ for $d \in \Re^{n_{d}}$, $c_{1}, \cdots, c_{n-1}, A, B, C, D$ are (unknown) constants or matrices, and $d:\left[t_{0}, \infty\right) \rightarrow \Gamma$ is a piecewise continuous function with its range $\Gamma$ a compact subset of $\Re^{n_{d}}$. It is noted that system (1.1) contains two classes of well known nonlinear systems as special cases. First, when $p_{i}=0$ for $i=1, \cdots, n$, system (1.1) becomes a subclass of the standard feedforward system studied in [10], and second, when $p_{i}=0$ for $i=2, \cdots, n$ and $p_{1} \neq 0$, system (1.1) becomes the feedforward system subject to input unmodeled dynamics as studied in [1]. Thus we can view system (1.1) as nonlinear systems in feedforward form subject to dynamic uncertainty $\xi_{1}, \xi_{2}, \cdots, \xi_{n}$ and static uncertainty $d$. System (1.1) is interesting on its own, on one hand, because dynamic uncertainty is ubiquitously present in real systems. On the other hand, it is known that the robust output regulation problem for a nonlinear system can be converted into a robust stabilization problem of an appropriately augmented system which can be viewed as the original system subject to dynamic and static uncertainties where the dynamic uncertainty models the internal model [2] and [3]. Thus the stabilization solution of system (1.1) also shed light on the solution of the robust output regulation problem of nonlinear systems in the standard feedforward form.

The objective of this paper is to design a static partial state feedback control law to semi-globally stabilize the

The work described in this paper was supported by a grant from the Research Grants Council of the Hong Kong Special Administration Region (Project no. CUHK412305). The authors are with the Department of Automation and Computer-Aided Engineering, The Chinese University of Hong Kong, Shatin, N. T., Hong Kong (e-mail: jhuang@acae.cuhk.edu.hk). equilibrium of the system. More precisely, given any compact subsets $0 \in X \subset \Re^{n}$ and $0 \in \Xi \subset \Re^{p}$ with $p=$ $p_{1}+p_{2} \cdots+p_{n}$, design a control law of the form $u=k(x)$ with $x=\operatorname{col}\left(x_{1}, x_{2}, \cdot, x_{n}\right)$ and $k(0)=0$, such that, for all $d$, the equilibrium of the closed-loop system is asymptotically stable with $X \times \Xi$ contained in the basin of attraction.

In addition to the fact that system (1.1) contains dynamic uncertainty, there is another difference between the above problem formulation and the one studied in [1] and [10] in that our formulation implies asymptotic disturbance rejection of time-varying exogenous signal $d$ in contrast with the disturbance attenuation in [10].

In comparison with the papers [1] and [10], a special difficulty of our problem arises from the fact that we will not assume that the Jacobian matrix of the closed-loop system at the origin can be made exponentially stable. As a result, we cannot employ the technique of the asymptotic small gain theorem. To overcome this difficulty, we will employ the technique of a small gain theorem with restrictions for uncertain time-varying nonlinear systems. This theorem can guarantee both the uniform stability and asymptotic gain properties with restrictions of two interconnected systems. An advantage of our problem formulation and technique is that we can handle a larger class of systems than those in [1]. Nevertheless, unlike asymptotic small gain theorem employed in [1] or [10], our small gain theorem does not guarantee that the interconnected system is ISS without restriction on the initial state even though the two subsystems are assumed to be ISS without restrictions on the initial states (but with restriction on the inputs). Therefore, our method does not have the capability of global asymptotic stabilization. The best we can do is semi-global stabilization and we have indeed achieved this goal.

Throughout the paper, let $L_{\infty}^{m}$ be the set of all piecewise continuous bounded functions $u:\left[t_{0}, \infty\right) \mapsto \Re^{m}$ with a finite supremum norm $\left\|u_{\left[t_{0}, \infty\right)}\right\|=\sup _{t \geq t_{0}}\|u(t)\|$, let $\left\|u_{\left[t_{1}, t_{2}\right]}\right\|=\sup _{t_{1} \leq t \leq t_{2}}\|u(t)\|$, and let $\|u\|_{a}=$ $\limsup \sup _{t \rightarrow \infty}\|u(t)\|$. Let $\bar{X} \subset \Re^{n}$ be a compact set containing the origin.

\section{SMALl GAIN THEOREM}

In this section, we will first introduce some terminologies adapted from [8] and [10] for time-varying nonlinear systems of the following form:

$$
\dot{x}=f(x, u, d, t), y=h(x, u, d, t), \quad t \geq t_{0} \geq 0
$$

where $x \in \Re^{n}$ is the plant state, $u \in \Re^{m}$ the input, $y \in \Re^{p}$ the output, the functions $f: \Re^{n} \times \Re^{m} \times \Re^{n_{d}} \times\left[t_{0}, \infty\right) \mapsto \Re^{n}$ and $h: \Re^{n} \times \Re^{m} \times \Re^{n_{d}} \times\left[t_{0}, \infty\right) \mapsto \Re^{p}$ are piecewise 
continuous in $\operatorname{col}(d, t)$ and locally Lipschitz in $\operatorname{col}(x, u)$ satisfying $f(0,0, d, t)=0$ and $h(0,0, d, t)=0$.

Definition 2.1: System (2.1) is said to satisfy robust asymptotic $L_{\infty}$ stability (RALS) with restrictions $X$ and $\Delta$ on the initial state $x\left(t_{0}\right)$ and the input $u$ respectively if for all $d$, there exist class $K$ functions $\gamma^{0}$ and $\gamma^{u}$ such that for any $x\left(t_{0}\right) \in X$ and any $u \in L_{\infty}^{m}$ satisfying $\left\|u_{\left[t_{0}, \infty\right)}\right\|<\Delta$, the solution of (2.1) exists and satisfies, for all $t \geq t_{0}$,

$$
\begin{aligned}
\|y(t)\| & \leq \max \left\{\gamma^{0}\left(\left\|x\left(t_{0}\right)\right\|\right), \gamma^{u}\left(\left\|u_{\left[t_{0}, \infty\right)}\right\|\right)\right\} \\
\|y\|_{a} & \leq \gamma^{u}\left(\|u\|_{a}\right)
\end{aligned}
$$

\section{-}

Remark 2.1: In the special case where $h(x, u, d, t)=x$, system (2.1) is said to be robust uniformly stable (RUS) with restrictions $X$ and $\Delta$ on the initial state $x\left(t_{0}\right)$ and the input $u$ if $y=x$ satisfies inequality (2.2) and is said to satisfy robust asymptotic gain (RAG) property with restrictions $X$ and $\Delta$ on the initial state $x\left(t_{0}\right)$ and the input $u$ if $y=$ $x$ satisfies inequality (2.3). It is known that a time-varying nonlinear system is US and has AG property with restriction $X$ on $x\left(t_{0}\right)$, then its equilibrium at the origin when $u=0$ is asymptotically stable with $X$ contained in the basin of attraction.

Consider the interconnection of the following two systems,

$$
\begin{aligned}
& \dot{x}_{1}=f_{1}\left(x_{1}, v_{1}, u_{1}, d, t\right), \quad y_{1}=h_{1}\left(x_{1}, v_{1}, u_{1}, d, t\right) \\
& \dot{x}_{2}=f_{2}\left(x_{2}, v_{2}, u_{2}, d, t\right), \quad y_{2}=h_{2}\left(x_{2}, v_{2}, u_{2}, d, t\right)
\end{aligned}
$$

subject to the interconnection:

$$
v_{1}=y_{2}, \quad v_{2}=y_{1}
$$

where, for $i=1,2, x_{i} \in \Re^{n_{i}}, u_{i} \in \Re^{m_{i}}, y_{i} \in$ $\Re^{p_{i}}, v_{i} \in \Re^{q_{i}}$ with $p_{1}=q_{2}, p_{2}=q_{1}$, the functions $f_{i}\left(x_{i}, v_{i}, u_{i}, d, t\right)$ and $h_{i}\left(x_{i}, v_{i}, u_{i}, d, t\right)$ are piecewise continuous in $\operatorname{col}(d, t)$ and locally Lipschitz in $\operatorname{col}\left(x_{i}, v_{i}, u_{i}\right)$ satisfying $f_{i}(0,0,0, d, t)=0$ and $h_{i}(0,0,0, d, t)=0$, and $d:\left[t_{0}, \infty\right) \mapsto \Re^{n_{d}}$ is piecewise continuous. Also, suppose the following.

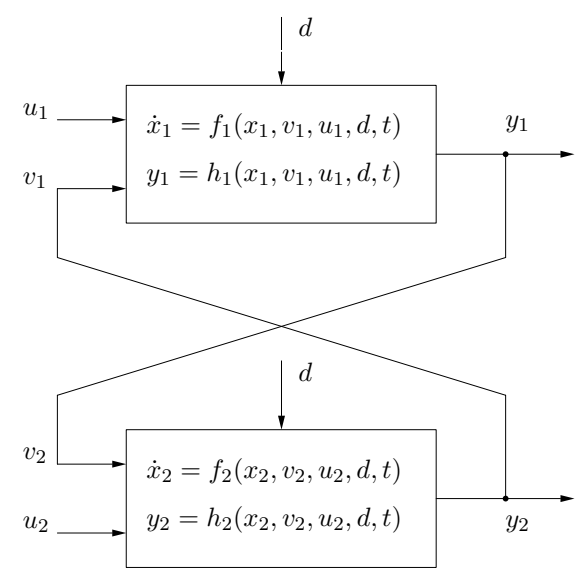

Fig. 1. Inter-connection of two subsystems
Assumption 2.1: The equations

$$
\begin{aligned}
& y_{1}=h_{1}\left(x_{1}, h_{2}\left(x_{2}, y_{1}, u_{2}, d, t\right), u_{1}, d, t\right) \\
& y_{2}=h_{2}\left(x_{2}, h_{1}\left(x_{1}, y_{2}, u_{1}, d, t\right), u_{2}, d, t\right)
\end{aligned}
$$

have a unique solution of the form $y=h(x, u, d, t)$ where $x=\operatorname{col}\left(x_{1}, x_{2}\right), y=\operatorname{col}\left(y_{1}, y_{2}\right), u=\operatorname{col}\left(u_{1}, u_{2}\right)$, and $h$ is locally Lipschitz in $\operatorname{col}(x, u)$ and piecewise continuous in $\operatorname{col}(d, t)$.

Theorem 2.1: Under Assumption 2.1, assume that both subsystems (2.4) and (2.5) are RUS and satisfy RAG and RALS with restrictions $X_{i}, \Delta_{i}$ and $\Delta_{i}^{u}$ on $x_{i}\left(t_{0}\right), v_{i}$ and $u_{i}$, $i=1,2$, respectively, i.e., for $i=1,2$, for all $d$, there exist class $K$ functions $\gamma_{i}^{0}, \gamma_{i}, \gamma_{i}^{u}, \bar{\gamma}_{i}^{0}, \bar{\gamma}_{i}$ and $\bar{\gamma}_{i}^{u}$, such that, for any $x_{i}\left(t_{0}\right) \in X_{i}, v_{i}(t) \in L_{\infty}^{q_{i}}$ satisfying $\left\|v_{i\left[t_{0}, \infty\right)}\right\|<\Delta_{i}$,

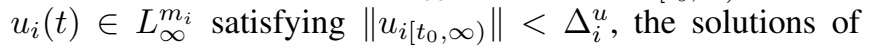
(2.4) and (2.5) exist and satisfy, for all $t \geq t_{0}$,

$$
\begin{aligned}
\left\|x_{i}(t)\right\| \leq & \max \left\{\gamma_{i}^{0}\left(\left\|x_{i}\left(t_{0}\right)\right\|\right)\right. \\
& \left.\gamma_{i}\left(\left\|v_{i\left[t_{0}, \infty\right)}\right\|\right), \gamma_{i}^{u}\left(\left\|u_{i\left[t_{0}, \infty\right)}\right\|\right)\right\} \\
\left\|x_{i}\right\|_{a} \leq & \max \left\{\gamma_{i}\left(\left\|v_{i}\right\|_{a}\right), \gamma_{i}^{u}\left(\left\|u_{i}\right\|_{a}\right)\right\} \\
\left\|y_{i}(t)\right\| \leq & \max \left\{\bar{\gamma}_{i}^{0}\left(\left\|x_{i}\left(t_{0}\right)\right\|\right)\right. \\
& \left.\bar{\gamma}_{i}\left(\left\|v_{i\left[t_{0}, \infty\right)}\right\|\right), \bar{\gamma}_{i}^{u}\left(\left\|u_{i\left[t_{0}, \infty\right)}\right\|\right)\right\} \\
\left\|y_{i}\right\|_{a} \leq & \max \left\{\bar{\gamma}_{i}\left(\left\|v_{i}\right\|_{a}\right), \bar{\gamma}_{i}^{u}\left(\left\|u_{i}\right\|_{a}\right)\right\}
\end{aligned}
$$

Suppose that the small gain condition

$$
\bar{\gamma}_{1} \circ \bar{\gamma}_{2}(r)<r, \quad r>0
$$

holds. Then, under connection (2.6), the system composed of (2.4) and (2.5) is RUS and satisfy RAG and RALS with restrictions $\tilde{X}_{1} \times \tilde{X}_{2}, \tilde{\Delta}_{1}$ and $\tilde{\Delta}_{2}$ on $x\left(t_{0}\right), u_{1}$ and $u_{2}$ respectively, viewing $x=\operatorname{col}\left(x_{1}, x_{2}\right)$ as state, $y=\operatorname{col}\left(y_{1}, y_{2}\right)$ as output and $u=\operatorname{col}\left(u_{1}, u_{2}\right)$ as input, i.e., for all $d$, there exist class $K$ functions $\gamma^{0}, \gamma^{u}, \bar{\gamma}^{0}$ and $\bar{\gamma}^{u}$, such that, for any initial state $x\left(t_{0}\right) \in \tilde{X}_{1} \times \tilde{X}_{2}$, and any input functions $u_{1}(t) \in L_{\infty}^{m_{1}}$ satisfying $\left\|u_{1\left[t_{0}, \infty\right)}\right\|<\tilde{\Delta}_{1}$ and $u_{2}(t) \in L_{\infty}^{m_{2}}$

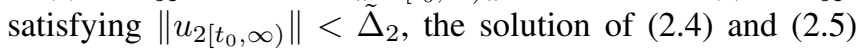
with connection (2.6) exists and satisfies, for all $t \geq t_{0}$,

$$
\begin{aligned}
\|x(t)\| & \leq \max \left\{\gamma^{0}\left(\left\|x\left(t_{0}\right)\right\|\right), \gamma^{u}\left(\left\|u_{\left[t_{0}, \infty\right)}\right\|\right)\right\} \\
\|x\|_{a} & \leq \gamma^{u}\left(\|u\|_{a}\right) \\
\|y(t)\| & \leq \max \left\{\bar{\gamma}^{0}\left(\left\|x\left(t_{0}\right)\right\|\right), \bar{\gamma}^{u}\left(\left\|u_{\left[t_{0}, \infty\right)}\right\|\right)\right\} \\
\|y\|_{a} & \leq \bar{\gamma}^{u}\left(\|u\|_{a}\right)
\end{aligned}
$$

where,

$$
\begin{aligned}
\gamma^{0}(s)= & \max \left\{2 \gamma_{1}^{0}(s), 2 \gamma_{1} \circ \bar{\gamma}_{2}^{0}(s), 2 \gamma_{1} \circ \bar{\gamma}_{2} \circ \bar{\gamma}_{1}^{0}(s),\right. \\
& \left.2 \gamma_{2}^{0}(s), 2 \gamma_{2} \circ \bar{\gamma}_{1}^{0}(s), 2 \gamma_{2} \circ \bar{\gamma}_{1} \circ \bar{\gamma}_{2}^{0}(s)\right\} \\
\gamma^{u}(s)= & \max \left\{2 \gamma_{1} \circ \bar{\gamma}_{2} \circ \bar{\gamma}_{1}^{u}(s), 2 \gamma_{1} \circ \bar{\gamma}_{2}^{u}(s), 2 \gamma_{1}^{u}(s),\right. \\
& \left.2 \gamma_{2} \circ \bar{\gamma}_{1} \circ \bar{\gamma}_{2}^{u}(s), 2 \gamma_{2} \circ \bar{\gamma}_{1}^{u}(s), 2 \gamma_{2}^{u}(s)\right\} \\
\bar{\gamma}^{0}(s)= & \max \left\{2 \bar{\gamma}_{1}^{0}(s), 2 \bar{\gamma}_{1} \circ \bar{\gamma}_{2}^{0}(s), 2 \bar{\gamma}_{2}^{0}(s), 2 \bar{\gamma}_{2} \circ \bar{\gamma}_{1}^{0}(s)\right\} \\
\bar{\gamma}^{u}(s)= & \max \left\{2 \bar{\gamma}_{1} \circ \bar{\gamma}_{2}^{u}(s), 2 \bar{\gamma}_{1}^{u}(s), 2 \bar{\gamma}_{2} \circ \bar{\gamma}_{1}^{u}(s), 2 \bar{\gamma}_{2}^{u}(s)\right\} \\
\tilde{X}_{1}= & \left\{x_{1} \in X_{1}: \bar{\gamma}_{1}^{0}\left(\left\|x_{1}\right\|\right)<\Delta_{2},\right. \\
& \left.\bar{\gamma}_{2} \circ \bar{\gamma}_{1}^{0}\left(\left\|x_{1}\right\|\right)<\Delta_{1}\right\} \\
\tilde{X}_{2}= & \left\{x_{2} \in X_{2}: \bar{\gamma}_{2}^{0}\left(\left\|x_{2}\right\|\right)<\Delta_{1},\right. \\
& \left.\bar{\gamma}_{1} \circ \bar{\gamma}_{2}^{0}\left(\left\|x_{2}\right\|\right)<\Delta_{2}\right\}
\end{aligned}
$$


and $\tilde{\Delta}_{1}$ is such that $\tilde{\Delta}_{1} \leq \Delta_{1}^{u}$, and

$s \in\left[0, \tilde{\Delta}_{1}\right) \Longrightarrow \bar{\gamma}_{2} \circ \bar{\gamma}_{1}^{u}(s)<\Delta_{1}, \bar{\gamma}_{1}^{u}(s)<\Delta_{2}$,

and $\tilde{\Delta}_{2}$ is such that $\tilde{\Delta}_{2} \leq \Delta_{2}^{u}$, and

$s \in\left[0, \tilde{\Delta}_{2}\right) \Longrightarrow \bar{\gamma}_{1} \circ \bar{\gamma}_{2}^{u}(s)<\Delta_{2}, \bar{\gamma}_{2}^{u}(s)<\Delta_{1}$.

Remark 2.2: This theorem is a slight extension of Theorem B.3.1 of [5] and the proof is thus omitted due to the space limit.

A special case of Theorem 2.1 which will be used in next section is when the second subsystem does not rely on $v_{2}$. In this case, $\bar{\gamma}_{2}$ in (2.9) can be any class $K$ function, thus making the small gain condition (2.11) always hold. Therefore, we have the following

Corollary 2.1: Consider the interconnection $v_{1}=y_{2}$ of the following two systems

$$
\begin{aligned}
& \dot{x}_{1}=f_{1}\left(x_{1}, v_{1}, u_{1}, d, t\right), \quad y_{1}=x_{1} \\
& \dot{x}_{2}=f_{2}\left(x_{2}, u_{2}, d, t\right), \quad y_{2}=x_{2} .
\end{aligned}
$$

Assume that subsystem (2.14) is RUS and satisfies RAG with restriction $X_{1}$ on $x_{1}\left(t_{0}\right)$ without restriction on $v_{1}$ and $u_{1}$, and that subsystem (2.15) is RUS and satisfies RAG with restrictions $X_{2}$ and $\Delta_{2}^{u}$ on $x_{2}\left(t_{0}\right)$ and $u_{2}$ respectively. Then system (2.14) and (2.15) with connection $v_{1}=y_{2}$ is RUS and satisfies RAG with restrictions $X_{1} \times X_{2}$ and $\Delta_{2}^{u}$ on $\left(x_{1}\left(t_{0}\right), x_{2}\left(t_{0}\right)\right)$ and $u_{2}$ respectively, i.e., for all $d$, there exist class $K$ functions $\gamma^{0}$ and $\gamma^{u}$, such that for any initial state $\left(x_{1}\left(t_{0}\right), x_{2}\left(t_{0}\right)\right) \in X_{1} \times X_{2}$, and any input functions $u_{1}(t) \in L_{\infty}^{m_{1}}$ and $u_{2}(t) \in L_{\infty}^{m_{2}}$ satisfying $\left\|u_{2\left[t_{0}, \infty\right)}\right\|<\Delta_{2}^{u}$, the solution of (2.14) and (2.15) with connection $v_{1}=y_{2}$ exists and satisfies, for all $t \geq t_{0}$,

$$
\begin{aligned}
\|x(t)\| & \leq \max \left\{\gamma^{0}\left(\left\|x\left(t_{0}\right)\right\|\right), \gamma^{u}\left(\left\|u_{\left[t_{0}, \infty\right)}\right\|\right)\right\} \\
\|x\|_{a} & \leq \gamma^{u}\left(\|u\|_{a}\right)
\end{aligned}
$$

where

$$
\begin{aligned}
\gamma^{0}(s) & =\max \left\{2 \gamma_{1}^{0}(s), 2 \gamma_{2}^{0}(s), 2 \gamma_{1} \circ \gamma_{2}^{0}(s)\right\} \\
\gamma^{u}(s) & =\max \left\{2 \gamma_{1}^{u}(s), 2 \gamma_{2}^{u}(s), 2 \gamma_{1} \circ \gamma_{2}^{u}(s)\right\}
\end{aligned}
$$

where all the gain functions are defined the same way as those in Theorem 2.1.

\section{MAIN RESULT}

Like [1], [10], our approach will utilize saturation functions characterized as follows.

Definition 3.1: A locally Lipschitz function $\sigma(\cdot): \Re \rightarrow$ $[-\lambda, \lambda]$ is said to be a saturation function with saturation level $\lambda>0$ if:

1) $\sigma(x)=x$ when $\|x\| \leq \frac{\lambda}{2}$

2) $\frac{\lambda}{2} \leq \operatorname{sgn}(x) \sigma(x) \leq \min \{\|x\|, \lambda\}$ when $\|x\| \geq \frac{\lambda}{2}$.

To state our assumptions, we rewrite, for $i=2, \cdots, n$,

$$
\begin{aligned}
& g_{i}\left(\xi_{1}, x_{1}, \ldots, \xi_{i-1}, x_{i-1}, \xi_{i}, u, d\right) \\
= & \tilde{g}_{i}\left(\xi_{1}, x_{1}, \ldots, \xi_{i-1}, x_{i-1}, \xi_{i}\right) \\
+ & h_{i}\left(\xi_{1}, x_{1}, \ldots, \xi_{i-1}, x_{i-1}, \xi_{i}, u, d\right)
\end{aligned}
$$

where $\tilde{g}_{i}\left(\xi_{1}, x_{1}, \ldots, \xi_{i-1}, x_{i-1}, \xi_{i}\right) \quad$ is linear in $\xi_{1}, x_{1}, \ldots, \xi_{i-1}, x_{i-1}, \xi_{i}$.
Assumption 3.1: For all $d \in \Gamma$, the following equations hold

$\lim _{\left\|\left(\xi_{1}, u\right)\right\| \rightarrow 0} \frac{\left\|f_{1}\left(\xi_{1}, u, d\right)\right\|}{\left\|\left(\xi_{1}, u\right)\right\|}=0, \lim _{\left\|\left(\xi_{1}, u\right)\right\| \rightarrow 0} \frac{\left\|g_{1}\left(\xi_{1}, u, d\right)\right\|}{\left\|\left(\xi_{1}, u\right)\right\|}=0$, and for $i=2, . ., n$,

$$
\begin{array}{r}
\lim _{\left\|\left(\xi_{1}, x_{1}, \ldots, \xi_{i}, u\right)\right\| \rightarrow 0} \frac{\left\|f_{i}\left(\xi_{1}, x_{1}, \ldots, \xi_{i}, u, d\right)\right\|}{\left\|\left(\xi_{1}, x_{1}, \ldots, \xi_{i}, u\right)\right\|}=0, \\
\lim _{\left\|\left(\xi_{1}, x_{1}, \ldots, \xi_{i}, u\right)\right\| \rightarrow 0} \frac{\left\|h_{i}\left(\xi_{1}, x_{1}, \ldots, \xi_{i}, u, d\right)\right\|}{\left\|\left(\xi_{1}, x_{1}, \ldots, \xi_{i}, u\right)\right\|}=0 .
\end{array}
$$

Assumption 3.2: For $i=1, \ldots, n-1, c_{i}^{L}<c_{i}<c_{i}^{H}$ for some positive numbers $c_{i}^{L}, c_{i}^{H}$. And the $d c$ gain $\vartheta^{L}<$ $\vartheta=D-C A^{-1} B<\vartheta^{H}$ for some positive numbers $\vartheta^{L}, \vartheta^{H}$, where $A$ is invertible.

Assumption 3.3: $\xi_{1}$ subsystem is RUS and satisfies RAG with a linear gain function viewing $\xi_{1}$ as state and $u$ as input. For $i=2, \ldots, n, \xi_{i}$ subsystem is RUS and satisfies RAG viewing $\xi_{i}$ as state and $\operatorname{col}\left(\xi_{1}, x_{1}, \ldots, \xi_{i-1}, x_{i-1}, u\right)$ as input.

Lemma 3.1: Consider the following system

$$
\begin{aligned}
\dot{x} & =C \xi+D u+G_{2}(\xi, u, d) \\
\dot{\xi} & =A \xi+B u+G_{1}(\xi, u, d)
\end{aligned}
$$

where, $x \in \Re, \xi \in \Re^{l}$, and, for $i=1,2$,

$$
\lim _{\|(\xi, u)\| \rightarrow 0} \frac{\left\|G_{i}(\xi, u, d)\right\|}{\|(\xi, u)\|}=0 .
$$

Assume the $d c$ gain $\vartheta^{L}<\vartheta=D-C A^{-1} B<\vartheta^{H}$ for positive numbers $\vartheta^{L}, \vartheta^{H}$. And the $\xi$ subsystem is RUS and satisfies RAG with restrictions $\Xi, \Delta_{u}$ on $\xi\left(t_{0}\right), u$ respectively and has a linear gain function, i.e., for all $d$, there exist class $K$ function $\gamma_{1}^{0}$ and positive numbers $N_{1}^{u}, \Delta_{u}$ such that for any $\xi\left(t_{0}\right) \in \Xi$ and any $u(t) \in L_{\infty}^{1}$ satisfying $\left\|u_{\left[t_{0}, \infty\right)}\right\|<$ $\Delta_{u}$, the solution $\xi(t)$ exists and satisfies, for all $t \geq t_{0}$,

$$
\begin{aligned}
\|\xi(t)\| & \leq \max \left\{\gamma_{1}^{0}\left(\left\|\xi\left(t_{0}\right)\right\|\right), N_{1}^{u}\left\|u_{\left[t_{0}, \infty\right)}\right\|\right\} \\
\|\xi\|_{a} & \leq N_{1}^{u}\|u\|_{a} .
\end{aligned}
$$

Then for any compact set $X \subset \Re$, there exists a control law $u=-\sigma(k x-\bar{u})$, where $\sigma$ is a saturation function with level $\lambda$, such that the system

$$
\dot{\bar{x}}=\left[\begin{array}{c}
\dot{x} \\
\dot{\xi}
\end{array}\right]=\left[\begin{array}{c}
C \xi+D u+G_{2}(\xi, u, d) \\
A \xi+B u+G_{1}(\xi, u, d)
\end{array}\right]_{u=-\sigma(k x-\bar{u})}
$$

is RUS and satisfies RAG with restrictions $X \times \Xi, \Delta_{\bar{u}}$ on $\bar{x}\left(t_{0}\right), \bar{u}$ respectively and a linear gain function, i.e., for all $d$, there exist class $K$ function $\gamma^{0}$ and positive numbers $N^{u}$, $\Delta_{\bar{u}}$ such that for any $\bar{x}\left(t_{0}\right) \in X \times \Xi$ and any $\bar{u}(t) \in L_{\infty}^{1}$ satisfying $\left\|\bar{u}_{\left[t_{0}, \infty\right)}\right\|<\Delta_{\bar{u}}$, the solution $\bar{x}(t)$ exists and satisfies, for all $t \geq t_{0}$

$$
\begin{aligned}
\|\bar{x}(t)\| & \leq \max \left\{\gamma^{0}\left(\left\|\bar{x}\left(t_{0}\right)\right\|\right), N^{u}\left\|\bar{u}_{\left[t_{0}, \infty\right)}\right\|\right\}, \\
\|\bar{x}\|_{a} & \leq N^{u}\|\bar{u}\|_{a} .
\end{aligned}
$$

Further, let $\left(\bar{A}_{1}, \bar{B}_{1}\right)$ be the Jacobian linearization of system (4.5), $\bar{C}_{1}=\left[\begin{array}{ll}c_{1} & 0_{(1 \times l)}\end{array}\right]$ with $c_{1}^{L}<c_{1}<c_{1}^{H}$ for some positive numbers $c_{1}^{L}, c_{1}^{H}$, and $\bar{D}_{1}=0$. Then, $\vartheta_{1}^{L}<\bar{\vartheta}_{1}=$ $\bar{D}_{1}-\bar{C}_{1} \bar{A}_{1}^{-1} \bar{B}_{1}<\vartheta_{1}^{H}$ for some positive numbers $\vartheta_{1}^{L}, \vartheta_{1}^{H}$. 
Remark 3.1: Lemma 3.1 is an extension of Lemma 1 of [1] in that it concerns both US and asymptotic gain property. Also, $d$ of Lemma 1 of [1] is treated as a disturbance to be attenuated while $d$ here is treated as a disturbance to be rejected.

Proof: The spirit of the proof is similar to that of the proof of Lemma 1 in [1]. That is, we need to employ the small gain theorem. For this purpose, introduce the same coordinate transformation $z=x-C A^{-1} \xi$ as in [1] to change system (4.5) into the following:

$$
\dot{\tilde{x}}=\left[\begin{array}{c}
\dot{z} \\
\dot{\xi}
\end{array}\right]=\left[\begin{array}{c}
\vartheta u+G(\xi, u, d) \\
A \xi+B u+G_{1}(\xi, u, d)
\end{array}\right]_{u=-\sigma(k x-\bar{u})}
$$

where $G(\xi, u, d)=G_{2}(\xi, u, d)-C A^{-1} G_{1}(\xi, u, d)$. Clearly,

$$
\lim _{\|(\xi, u)\| \rightarrow 0} \frac{\|G(\xi, u, d)\|}{\|(\xi, u)\|}=0 .
$$

Define $\tilde{\lambda}=\vartheta \lambda$ and $\tilde{k}=\vartheta k$. Then

$$
\vartheta u=-\vartheta \sigma\left(k\left(x-\frac{\bar{u}}{k}\right)\right)=-\tilde{\sigma}\left(\tilde{k}\left(z+C A^{-1} \xi-\frac{\bar{u}}{k}\right)\right)
$$

where $\tilde{\sigma}(s)=\vartheta \sigma(s / \vartheta)$ is a saturation function with level $\tilde{\lambda}$.

With (4.8), system (4.6) can be viewed as the interconnection

$$
v_{1}=y_{2}, \quad v_{2}=\left[\begin{array}{l}
v_{21} \\
v_{22}
\end{array}\right]=y_{1}
$$

of the following two subsystems

$$
\begin{aligned}
\Sigma_{1}: \quad \dot{\xi} & =A \xi-B \sigma\left(k v_{1}\right)+G_{1}\left(\xi,-\sigma\left(k v_{1}\right), d\right), \\
y_{1} & =\left[\begin{array}{l}
y_{11} \\
y_{12}
\end{array}\right]=\left[\begin{array}{c}
C A^{-1} \xi \\
G\left(\xi,-\sigma\left(k v_{1}\right), d\right) / \tilde{k}
\end{array}\right], \\
\Sigma_{2}: \quad \dot{z} & =-\tilde{\sigma}\left(\tilde{k}\left(z+v_{21}-\frac{\bar{u}}{k}\right)\right)+\tilde{k} v_{22}, \\
y_{2} & =z+v_{21}-\frac{\bar{u}}{k} .
\end{aligned}
$$

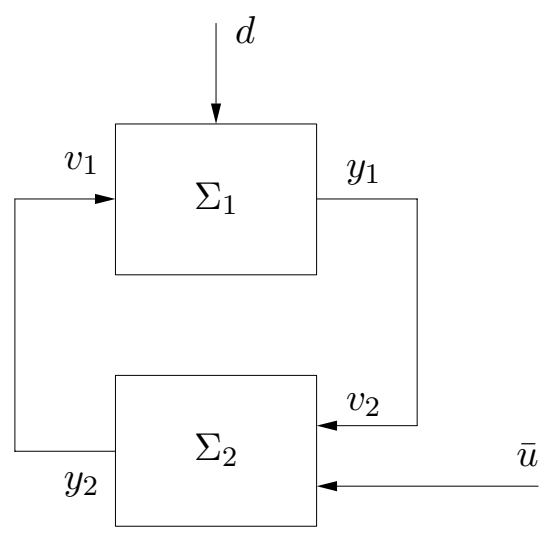

Fig. 2. Inter-connection of $\Sigma_{1}$ and $\Sigma_{2}$

We will now apply Theorem 2.1 to show that system (4.5) is RUS and satisfies RAG with appropriate restrictions in four steps.
Step 1. Show subsystem $\Sigma_{2}$ is ISS and satisfies ALS with no restrictions on $z\left(t_{0}\right)$ and $\bar{u}$ and with restriction $\Delta_{2}=$ $\lambda /(2 k)$ on $v_{2}$. Let $V(z)=z^{2} / 2$. Then its derivative along the trajectory of $\Sigma_{2}$ satisfies

$$
\dot{V}=-\left(\tilde{\sigma}\left(\tilde{k}\left(z+v_{21}-\frac{\bar{u}}{k}\right)\right)-\tilde{k} v_{22}\right) z
$$

It can be shown that under the restriction $\left\|v_{22\left[t_{0}, \infty\right)}\right\|<$ $\tilde{\lambda} /(2 \tilde{k})=\lambda /(2 k)$, the following implication holds

$$
\|z\|>\max \left\{3\left\|v_{21}\right\|, 3\left\|\frac{\bar{u}}{k}\right\|, 3\left\|v_{22}\right\|\right\} \Rightarrow \dot{V}<0 .
$$

Hence, by Lemma $3.3[10], V(z)=z^{2} / 2$ is a local ISS Lyapunov function for subsystem $\Sigma_{2}$. Therefore, for all $d$, there exists class $K$ function $\gamma_{2}^{0}$ such that for any $z\left(t_{0}\right) \in \Re$, and any $v_{22}(t) \in L_{\infty}^{1}$ satisfying $\left\|v_{22\left[t_{0}, \infty\right)}\right\|<\tilde{\lambda} /(2 \tilde{k})=$ $\lambda /(2 k), v_{21}(t) \in L_{\infty}^{1}$ and $\bar{u}(t) \in L_{\infty}^{1}$, the solution $z(t)$ exists for all $t \geq t_{0}$ and satisfies

$$
\begin{aligned}
\|z(t)\| \leq & \max \left\{\gamma_{2}^{0}\left(\left\|z\left(t_{0}\right)\right\|\right), 3\left\|v_{21\left[t_{0}, \infty\right)}\right\|,\right. \\
& \left.3\left\|v_{22\left[t_{0}, \infty\right)}\right\|, \frac{3}{k}\left\|\bar{u}_{\left[t_{0}, \infty\right)}\right\|\right\} \\
\|z\|_{a} \leq & \max \left\{3\left\|v_{21}\right\|_{a}, 3\left\|v_{22}\right\|_{a}, \frac{3}{k}\|\bar{u}\|_{a}\right\} .
\end{aligned}
$$

It follows from (4.10) and $y_{2}=z+v_{21}-\frac{\bar{u}}{k}$ that for any $z\left(t_{0}\right) \in \Re$, and any $v_{22}(t) \in L_{\infty}^{1}$ satisfying $\left\|v_{22\left[t_{0}, \infty\right)}\right\|<$ $\tilde{\lambda} /(2 \tilde{k})=\lambda /(2 k), v_{21}(t) \in L_{\infty}^{1}$ and $\bar{u}(t) \in L_{\infty}^{1}$, the output $y_{2}(t)$ exists for all $t \geq t_{0}$ and satisfies

$$
\begin{aligned}
\left\|y_{2}(t)\right\| \leq & \max \left\{\bar{\gamma}_{2}^{0}\left(\left\|z\left(t_{0}\right)\right\|\right), 9\left\|v_{21\left[t_{0}, \infty\right)}\right\|,\right. \\
& \left.9\left\|v_{22\left[t_{0}, \infty\right)}\right\|, \frac{9}{k}\left\|\bar{u}_{\left[t_{0}, \infty\right)}\right\|\right\} \\
\leq & \max \left\{\bar{\gamma}_{2}^{0}\left(\left\|z\left(t_{0}\right)\right\|\right), \bar{\gamma}_{2}\left(\left\|v_{2\left[t_{0}, \infty\right)}\right\|\right)\right. \\
& \left.\bar{\gamma}_{2}^{u}\left(\left\|\bar{u}_{\left[t_{0}, \infty\right)}\right\|\right)\right\} \\
\left\|y_{2}\right\|_{a} \leq & \max \left\{9\left\|v_{21}\right\|_{a}, 9\left\|v_{22}\right\|_{a}, \frac{9}{k}\|\bar{u}\|_{a}\right\} \\
\leq & \max \left\{\bar{\gamma}_{2}\left(\left\|v_{2}\right\|_{a}\right), \bar{\gamma}_{2}^{u}\left(\|\bar{u}\|_{a}\right\}\right)
\end{aligned}
$$

where $\bar{\gamma}_{2}^{0}(s)=3 \gamma_{2}^{0}(s)$ and $\bar{\gamma}_{2}(s)=9 s$. Obviously, $\Sigma_{2}$ is ISS and ALS with restriction $\Delta_{2}=\lambda /(2 k)$ on $v_{2}$.

Step 2. Show subsystem $\Sigma_{1}$ is RUS and satisfies RAG and RALS with restriction $\Xi$ on $\xi\left(t_{0}\right)$ and without restriction on $v_{1}$. Choose $\lambda$ such that $\lambda<\Delta_{u}$ to guarantee $\left\|u_{\left[t_{0}, \infty\right)}\right\|<$ $\Delta_{u}$. Noting

$$
\left\|\sigma\left(k v_{1}\right)\right\| \leq \min \left\{k\left\|v_{1}\right\|, \lambda\right\} \leq k\left\|v_{1}\right\|
$$

and substituting (4.12) into (4.4) gives that for any $\xi\left(t_{0}\right) \in \Xi$ and any $v_{1}(t) \in L_{\infty}^{1}$, the solution $\xi(t)$ exists for all $t \geq t_{0}$ and satisfies

$$
\begin{aligned}
\|\xi(t)\| & \leq \max \left\{\gamma_{1}^{0}\left(\left\|\xi\left(t_{0}\right)\right\|\right), N_{1}^{u}\left\|\sigma\left(k v_{1\left[t_{0}, \infty\right)}\right)\right\|\right\} \\
& \leq \max \left\{\gamma_{1}^{0}\left(\left\|\xi\left(t_{0}\right)\right\|\right), N_{1}^{u} k\left\|v_{1\left[t_{0}, \infty\right)}\right\|\right\} \\
\|\xi\|_{a} & \leq N_{1}^{u} k\left\|v_{1}\right\|_{a} .
\end{aligned}
$$

Let $l$ be a positive number such that $\left\|C A^{-1}\right\|<l$. Then, for any $\xi\left(t_{0}\right) \in \Xi$ and any $v_{1}(t) \in L_{\infty}^{1}$, the solution $\xi(t)$ exists 
for all $t \geq t_{0}$ and satisfies

$$
\begin{aligned}
\left\|y_{11}(t)\right\| & =\left\|C A^{-1} \xi(t)\right\| \\
& \leq \max \left\{\bar{\gamma}_{11}^{0}\left(\left\|\xi\left(t_{0}\right)\right\|\right), l N_{1}^{u} k\left\|v_{1\left[t_{0}, \infty\right)}\right\|\right\} \\
& =\max \left\{\bar{\gamma}_{11}^{0}\left(\left\|\xi\left(t_{0}\right)\right\|\right), \bar{\gamma}_{11}\left(\left\|v_{1\left[t_{0}, \infty\right)}\right\|\right)\right\} \\
\left\|y_{11}\right\|_{a} & =\left\|C A^{-1} \xi\right\|_{a} \leq \bar{\gamma}_{11}\left(\left\|v_{1}\right\|_{a}\right)
\end{aligned}
$$

where $\bar{\gamma}_{11}^{0}(s)=l \gamma_{1}^{0}(s)$.

Next consider $y_{12}$. It follows from (4.7) that there exists a nondecreasing, continuous function $\gamma_{o}(s)$ such that

$$
\left\|G\left(\xi,-\sigma\left(k v_{1}\right), d\right)\right\| \leq \max \left\{\gamma_{o}(\|\xi\|), \gamma_{o}\left(\left\|\sigma\left(k v_{1}\right)\right\|\right)\right\} .
$$

where

$$
\lim _{\|s\| \rightarrow 0} \frac{\left\|\gamma_{o}(s)\right\|}{\|s\|}=0 .
$$

Using (4.4) and (4.12) gives that for any $\xi\left(t_{0}\right) \in \Xi$ and any $v_{1}(t) \in L_{\infty}^{1}$, the following estimates hold

$$
\begin{aligned}
\left\|y_{12}(t)\right\| \leq & \max \left\{\gamma_{o} \circ \gamma_{1}^{0}\left(\left\|\xi\left(t_{0}\right)\right\|\right) / \tilde{k},\right. \\
& \gamma_{o}\left(N_{1}^{u}\left\|\sigma\left(k v_{1\left[t_{0}, \infty\right)}\right)\right\|\right) / \tilde{k}, \\
& \left.\gamma_{o}\left(\left\|\sigma\left(k v_{1\left[t_{0}, \infty\right)}\right)\right\|\right) / \tilde{k}\right\} \\
\leq & \max \left\{\bar{\gamma}_{12}^{0}\left(\left\|\xi\left(t_{0}\right)\right\|\right), \bar{\gamma}_{o}\left(\left\|\sigma\left(k v_{1\left[t_{0}, \infty\right)}\right)\right\|\right) / \tilde{k}\right\} \\
\leq & \max \left\{\bar{\gamma}_{12}^{0}\left(\left\|\xi\left(t_{0}\right)\right\|\right), \bar{\gamma}_{12}\left(\left\|v_{1\left[t_{0}, \infty\right)}\right\|\right)\right\}, \\
\left\|y_{12}\right\|_{a} \leq & \max \left\{\gamma_{o}\left(N_{1}^{u}\left\|\sigma\left(k v_{1}\right)\right\|_{a}\right) / \tilde{k}, \gamma_{o}\left(\left\|\sigma\left(k v_{1}\right)\right\|_{a}\right) / \tilde{k}\right\} \\
\leq & \bar{\gamma}_{o}\left(\left\|\sigma\left(k v_{1}\right)\right\|_{a}\right) / \tilde{k} \leq \bar{\gamma}_{12}\left(\left\|v_{1}\right\|_{a}\right)
\end{aligned}
$$

where $\bar{\gamma}_{12}^{0}(s)=\gamma_{o} \circ \gamma_{1}^{0}(s) / \tilde{k}, \quad \bar{\gamma}_{o}(s)=$ $\max \left\{\gamma_{o}\left(N_{1}^{u} s\right), \gamma_{o}(s)\right\}$, and $\bar{\gamma}_{12}(s)=\bar{\gamma}_{o}(\min \{k s, \lambda\}) / \tilde{k}$. The gain function $\bar{\gamma}_{12}(s)$ can be written as follows:

$$
\bar{\gamma}_{12}(s)=\left\{\begin{array}{cc}
\frac{\bar{\gamma}_{o}(k s)}{\vartheta k}, & 0<s \leq \frac{\lambda}{k} \\
\frac{\bar{\gamma}_{o}(\lambda)}{\vartheta k}, & s \geq \frac{\lambda}{k}
\end{array}\right.
$$

Due to (4.15), for any $\epsilon>0$, there exists $\delta>0$ such that

$$
\bar{\gamma}_{o}(s)<\epsilon s, 0<s \leq \delta .
$$

Thus, letting $\lambda=\delta$ gives

$$
\bar{\gamma}_{12}(s)<\frac{\epsilon}{\vartheta} s, s>0
$$

Combining (4.14), (4.16) and (4.18) gives that for any $\xi\left(t_{0}\right) \in \Xi$ and any $v_{1}(t) \in L_{\infty}^{1}$, the following estimates hold

$$
\begin{aligned}
\left\|y_{1}(t)\right\| & \leq \max \left\{\bar{\gamma}_{1}^{0}\left(\left\|\xi\left(t_{0}\right)\right\|\right), \bar{\gamma}_{1}\left(\left\|v_{1\left[t_{0}, \infty\right)}\right\|\right)\right\} \\
\left\|y_{1}\right\|_{a} & \leq \bar{\gamma}_{1}\left(\left\|v_{1}\right\|_{a}\right),
\end{aligned}
$$

where $\bar{\gamma}_{1}(s)=\left(l N_{1}^{u} k+\frac{\epsilon}{\vartheta}\right) s, \bar{\gamma}_{1}^{0}(s)=\bar{\gamma}_{11}^{0}(s)+\bar{\gamma}_{12}^{0}(s)$.

Step 3. Choose $k$ and $\epsilon$ to satisfy the small gain condition. For this purpose, note that

$$
\bar{\gamma}_{1} \circ \bar{\gamma}_{2}(s)=9\left(l N_{1}^{u} k+\frac{\epsilon}{\vartheta}\right) s .
$$

It suffices to choose $k$ and $\epsilon$ sufficiently small such that

$$
9\left(l N_{1}^{u} k+\frac{\epsilon}{\vartheta^{L}}\right)<1 .
$$

Note that $\lambda$ is determined by $\epsilon$ and is independent of $k$. Therefore, it is possible to choose $k$ and $\lambda$ such that the small gain condition is satisfied while $\frac{\lambda}{k}$ is sufficiently large.
By Theorem 2.1, system (4.6) is RUS and satisfies RAG with restrictions $\tilde{X} \times \tilde{\Xi}, \Delta_{\bar{u}}$ on $\tilde{x}\left(t_{0}\right), \bar{u}$ respectively, viewing $\tilde{x}=\operatorname{col}(z, \xi)$ as state and $\bar{u}$ as input, i.e., for all $d$, there exist class $K$ function $\tilde{\gamma}^{0}$ and positive numbers $\tilde{N}^{u}$ and $\Delta_{\bar{u}}$, such that, for any $\tilde{x}\left(t_{0}\right) \in \tilde{X} \times \tilde{\Xi}$, and $\bar{u}(t) \in L_{\infty}^{1}$ satisfying $\left\|\bar{u}_{\left[t_{0}, \infty\right)}\right\|<\Delta_{\bar{u}}$, the solution of $\Sigma_{1}$ and $\Sigma_{2}$ with connection (4.9) exists and satisfies, for all $t \geq t_{0}$,

$$
\begin{aligned}
\|\tilde{x}(t)\| & \leq \max \left\{\tilde{\gamma}^{0}\left(\left\|\tilde{x}\left(t_{0}\right)\right\|\right), \tilde{N}^{u}\left\|\bar{u}_{\left[t_{0}, \infty\right)}\right\|\right\} \\
\|\tilde{x}\|_{a} & \leq \tilde{N}^{u}\|\bar{u}\|_{a}
\end{aligned}
$$

where,

$\tilde{\gamma}^{0}(s)=\max \left\{2 \gamma_{1}^{0}(s), 2 N_{1}^{u} k \bar{\gamma}_{2}^{0}(s), 18 N_{1}^{u} k \bar{\gamma}_{1}^{0}(s), 2 \gamma_{2}^{0}(s)\right.$,

$\left.6 \bar{\gamma}_{1}^{0}(s), 6\left(l N_{1}^{u} k+\frac{\epsilon}{\vartheta L}\right) \bar{\gamma}_{2}^{0}(s)\right\}$,

$\tilde{N}^{u}=\max \left\{18 N_{1}^{u}, 54\left(l N_{1}^{u} k+\frac{\epsilon}{\vartheta^{L}}\right) / k, 6 / k\right\}$, and

$\tilde{\Xi}=\left\{\xi \in \Xi: \bar{\gamma}_{1}^{0}(\|\xi\|)<\lambda /(2 k)\right\}$,

$\tilde{X}=\left\{z \in \Re:\left(l N_{1}^{u} k+\frac{\epsilon}{\vartheta^{L}}\right) \bar{\gamma}_{2}^{0}(\|z\|)<\lambda /(2 k)\right\}$, and $\Delta_{\bar{u}}=\lambda /\left(18\left(l N_{1}^{u} k+\frac{\epsilon}{\vartheta^{L}}\right)\right)$

Step 4. Since $C A^{-1} \xi=x-z$, for any $\tilde{x}\left(t_{0}\right) \in \tilde{X} \times \tilde{\Xi}$, and $\bar{u}(t) \in L_{\infty}^{1}$ satisfying $\left\|\bar{u}_{\left[t_{0}, \infty\right)}\right\|<\Delta_{\bar{u}}$, the solution of system (4.5) exists and satisfies, for all $t \geq t_{0}$,

$$
\begin{aligned}
\|\bar{x}(t)\| & \leq \max \{2\|\xi(t)\|, 2\|x(t)\|\} \\
& =\max \left\{2\|\xi(t)\|, 2\left\|\left(z+C A^{-1} \xi\right)(t)\right\|\right\} \\
& \leq 4(1+l) \max \{\|z(t)\|,\|\xi(t)\|\} \\
& \leq 4(1+l)\|\tilde{x}(t)\| \\
& \leq \max \left\{\gamma^{0}\left(\left\|\bar{x}\left(t_{0}\right)\right\|\right), N^{u}\left\|\bar{u}_{\left[t_{0}, \infty\right)}\right\|\right\}
\end{aligned}
$$

where $\gamma^{0}(s)=4(1+l) \tilde{\gamma}^{0}(4(1+l) s)$ and $N^{u}=4(1+l) \tilde{N}^{u}$.

Similarly, it holds that

$$
\|\bar{x}\|_{a} \leq N^{u}\|\bar{u}\|_{a} .
$$

Since $\frac{\lambda}{k}$ can be arbitrarily large so that $\tilde{\Xi}=\Xi$ and $X \subset$ $\tilde{X}$, system (4.5) is RUS and satisfies RAG with restrictions $X \times \Xi, \Delta_{\bar{u}}$ on $\bar{x}\left(t_{0}\right), \bar{u}$ and has a linear gain function $N^{u} s$, viewing $\bar{u}$ as input, $\bar{x}=\operatorname{col}(x, \xi)$ as state.

Finally, note that the Jacobian linearization of (4.5) at the origin is

$$
\bar{A}_{1}=\left[\begin{array}{cc}
-k D & C \\
-k B & A
\end{array}\right], \quad \bar{B}_{1}=\left[\begin{array}{l}
D \\
B
\end{array}\right] .
$$

Using $\bar{C}_{1}=\left[\begin{array}{ll}c_{1} & 0_{(1 \times l)}\end{array}\right], \bar{D}_{1}=0$ gives that $\vartheta_{1}^{L}=\frac{c_{1}^{L}}{k}<$ $\bar{\vartheta}_{1}=\bar{D}_{1}-\bar{C}_{1} \bar{A}_{1}^{-1} \bar{B}_{1}=\frac{c_{1}}{k}<\frac{c_{1}^{H}}{k}=\vartheta_{1}^{H}$.

Lemma 3.2: Consider the following system

$$
\begin{aligned}
\dot{\zeta} & =G_{3}(\zeta, x, \xi)+H_{3}(\zeta, x, \xi, u, d) \\
\dot{x} & =C \xi+D u+G_{2}(\xi, u, d) \\
\dot{\xi} & =A \xi+B u+G_{1}(\xi, u, d)
\end{aligned}
$$

where, $x \in \Re, \xi \in \Re^{l}, \zeta \in \Re^{p}, G_{3}$ is linear in $\zeta, x, \xi$, and

$$
\begin{aligned}
\lim _{\|(\xi, u)\| \rightarrow 0} \frac{\left\|G_{i}(\xi, u, d)\right\|}{\|(\xi, u)\|}=0, \quad i=1,2, \\
\lim _{\|(\zeta, x, \xi, u)\| \rightarrow 0} \frac{\left\|H_{3}(\zeta, x, \xi, u, d)\right\|}{\|(\zeta, x, \xi, u)\|}=0 .
\end{aligned}
$$

Assume that $(x, \xi)$ subsystem satisfies all assumptions in Lemma 3.1. Moreover, $\zeta$ subsystem is RUS and satisfies 
RAG with a linear gain function viewing $\zeta$ as state and $\operatorname{col}(x, \xi, u)$ as input. Then for any compact sets $Z \subset \Re^{p}$ and $X \subset \Re$, there exists a control law $u=-\sigma(k x-\bar{u})$, where $\sigma$ is a saturation function with level $\lambda$ such that the system

$$
\begin{aligned}
\dot{\bar{\zeta}} & =\left[\begin{array}{c}
\dot{\zeta} \\
\dot{x} \\
\dot{\xi}
\end{array}\right] \\
= & {\left[\begin{array}{c}
G_{3}(\zeta, x, \xi)+H_{3}(\zeta, x, \xi, u, d) \\
C \xi+D u+G_{2}(\xi, u, d) \\
A \xi+B u+G_{1}(\xi, u, d)
\end{array}\right]_{u=-\sigma(k x-\bar{u})} }
\end{aligned}
$$

is RUS and satisfies RAG with restrictions $Z \times X \times \Xi$, $\Delta_{\bar{u}}$ on $\bar{\zeta}\left(t_{0}\right), \bar{u}$ respectively and has a linear gain function. Moreover, $\vartheta_{1}^{L}<\vartheta_{1}=D_{1}-C_{1} A_{1}^{-1} B_{1}<\vartheta_{1}^{H}$ for positive numbers $\vartheta_{1}^{L}, \vartheta_{1}^{H}$, where $\left(A_{1}, B_{1}\right)$ is the Jacobian linearization of system (4.25) and $C_{1}=\left[\begin{array}{ll}0_{(1 \times p)} & \bar{C}_{1}\end{array}\right], D_{1}=0$.

Proof: It follows from Lemma 3.1 that for any compact set $X \subset \Re$, there exists a control law $u=-\sigma(k x-\bar{u})$, where $\sigma$ is a saturation function with level $\lambda$, such that the system

$$
\dot{\bar{x}}=\left[\begin{array}{c}
\dot{x} \\
\dot{\xi}
\end{array}\right]=\left[\begin{array}{c}
C \xi+D u+G_{2}(\xi, u, d) \\
A \xi+B u+G_{1}(\xi, u, d)
\end{array}\right]_{u=-\sigma(k x-\bar{u})}
$$

is RUS and satisfies RAG with restrictions $X \times \Xi, \Delta_{\bar{u}}$ on $\bar{x}\left(t_{0}\right), \bar{u}$ respectively and has a linear gain function. And $\bar{\vartheta}_{1}=\bar{D}_{1}-\bar{C}_{1} \bar{A}_{1}^{-1} \bar{B}_{1}=\frac{c_{1}}{k}$ with $c_{1}^{L}<c_{1}<c_{1}^{H}$ for some positive numbers $c_{1}^{L}, c_{1}^{H}$, where $\left(\bar{A}_{1}, \bar{B}_{1}\right)$ is the Jacobian linearization of system (4.26) at the origin and $\bar{C}_{1}=\left[\begin{array}{ll}c_{1} & 0_{(1 \times l)}\end{array}\right], \bar{D}_{1}=0$.

Since $\zeta$ subsystem is RUS and satisfies RAG with a linear gain function viewing $\zeta$ as state and $\operatorname{col}(x, \xi, u)$ as input, i.e., for all $d$, there exist positive numbers $N_{x}, N_{\xi}, N_{u}$ and class $K$ function $\gamma^{0}(s)$ such that for any $\zeta\left(t_{0}\right) \in \Re^{p}$, the following estimates hold for all $t \geq t_{0}$,

$$
\begin{aligned}
\|\zeta(t)\| \leq & \max \left\{\gamma^{0}\left(\left\|\zeta\left(t_{0}\right)\right\|\right), N_{x}\left\|x_{\left[t_{0}, \infty\right)}\right\|\right. \\
& \left.N_{\xi}\left\|\xi_{\left[t_{0}, \infty\right)}\right\|, N_{u}\left\|u_{\left[t_{0}, \infty\right)}\right\|\right\} \\
\|\zeta\|_{a} \leq & \max \left\{N_{x}\|x\|_{a}, N_{\xi}\|\xi\|_{a}, N_{u}\|u\|_{a}\right\}
\end{aligned}
$$

Note that

$$
\begin{aligned}
\left\|u_{\left[t_{0}, \infty\right)}\right\| & \leq\left\|(k x-\bar{u})_{\left[t_{0}, \infty\right)}\right\| \\
& \leq \max \left\{2 k\left\|x_{\left[t_{0}, \infty\right)}\right\|, 2\left\|\bar{u}_{\left[t_{0}, \infty\right)}\right\|\right\} \\
\|u\|_{a} & \leq\|k x-\bar{u}\|_{a} \\
& \leq \max \left\{2 k\|x\|_{a}, 2\|\bar{u}\|_{a}\right\} .
\end{aligned}
$$

Substituting (4.28) into (4.27) gives that

$$
\begin{aligned}
\|\zeta(t)\| \leq & \max \left\{\gamma^{0}\left(\left\|\zeta\left(t_{0}\right)\right\|\right), N_{x}\left\|x_{\left[t_{0}, \infty\right)}\right\|, N_{\xi}\left\|\xi_{\left[t_{0}, \infty\right)}\right\|,\right. \\
& \left.2 k N_{u}\left\|x_{\left[t_{0}, \infty\right)}\right\|, 2 N_{u}\left\|\bar{u}_{\left[t_{0}, \infty\right)}\right\|\right\} \\
\|\zeta\|_{a} \leq & \max \left\{N_{x}\|x\|_{a}, N_{\xi}\|\xi\|_{a}, 2 k N_{u}\|x\|_{a}, 2 N_{u}\|\bar{u}\|_{a}\right\} .
\end{aligned}
$$

Hence, $\zeta$ subsystem is RUS and satisfies RAG with a linear gain function viewing $\zeta$ as state and $\operatorname{col}(x, \xi, \bar{u})$ as input. We invoke Corollary 2.1 to conclude that for any compact set $Z \subset \Re^{p}, \bar{\zeta}$ system is RUS and satisfies RAG with a linear gain function and restrictions $Z \times X \times \Xi$ and $\Delta_{\bar{u}}$ on $\bar{\zeta}\left(t_{0}\right)$ and $\bar{u}$ respectively.

The Jacobian linearization of system (4.25) at the origin is in the form:

$$
A_{1}=\left[\begin{array}{cc}
* & * \\
0 & \bar{A}_{1}
\end{array}\right], \quad B_{1}=\left[\begin{array}{c}
0 \\
\bar{B}_{1}
\end{array}\right]
$$

with $\bar{A}_{1}, \bar{B}_{1}$ being given by (4.22). Therefore,

$$
\frac{c_{1}^{L}}{k}<\vartheta_{1}=D_{1}-C_{1} A_{1}^{-1} B_{1}=\bar{D}_{1}-\bar{C}_{1} \bar{A}_{1}^{-1} \bar{B}_{1}<\frac{c_{1}^{H}}{k} \text {. }
$$

Using Lemmas 3.1 and 3.2, it is possible to establish the following result.

Theorem 3.1: Consider system (1.1), under Assumptions 3.1-3.3, there exists a control law of the form $u=$ $-\sigma_{1}\left(k_{1} x_{1}+\sigma_{2}\left(k_{2} x_{2}+\cdots+\sigma_{n}\left(k_{n} x_{n}\right)\right)\right)$ that solves the semi-global robust stabilization problem for system (1.1).

Proof: The proof can be obtained by recursive applications of Lemma 3.1 and Lemma 3.2.

\section{REFERENCES}

[1] M. Arcak, A. R. Teel and P. Kokotovic, Robust nonlinear control of feedforward systems with unmodeled dynamics, Automatica, vol. 37 , pp. 265-272, 2001.

[2] J. Huang and Z. Chen, A general framework for tackling the output regulation problem, IEEE Transactions on Automatic Control, vol. 49, pp. 2203-2218, 2004.

[3] J. Huang, Nonlinear output regulation: Theory and applications, SIAM, Philadelphia, 2004.

[4] A. Isidori, Nonlinear control systems, New York: Springer-Verlag, vol. II, 1999.

[5] A. Isidori, L. Marconi, and A. Serrani, Robust autonomous guidance: An internal model approach, New York: Springer, 2003.

[6] Z.P. Jiang, A.R. Teel and L. Praly, Small-gain theorem for ISS sysems and applications, Mathematics of Control, Signals and Systems, vol. 7, pp. 95-120, 1994.

[7] E.D. Sontag, Smooth stabilization implies coprime factorization, IEEE Transactions on Automatic Control, vol. 34, pp. 435-443, 1989.

[8] E.D. Sontag and Y. Wang, New characterization of input-to-state stability, IEEE Transactions on Automatic Control, vol. 41, pp. 12831294, 1996.

[9] E.D. Sontag and Y. Wang, On the characteristics of the Input-to-State Stability Property, Systems and Control Letters, vol. 24, pp. 351-359, 1995.

[10] A.R. Teel, A nonlinear small gain theorem for the analysis of control systems with saturation, IEEE Transactions on Automatic Control, vol. 41, pp. 1256-1270, 1996 